\title{
A Legislation-Oriented VLE-MAS System Applied to MOODLE
}

\author{
Maria Isabel Giusti Moreira ${ }^{1}$, Marilton Sanchotene de Aguiar ${ }^{2}$, Antônio Carlos Da \\ Rocha Costa ${ }^{1}$, Verlani Tim Hinz ${ }^{1}$ \\ ${ }^{1}$ Universidade Federal do Rio Grande do Sul (UFRGS), \\ Brasil \\ ${ }^{2}$ Universidade Federal de Pelotas (UFPel), \\ Brasil
}

\{isabelmoreira, vertimm, ac.rocha.costa\}@gmail.com, marilton@inf.ufpel.edu.br

\begin{abstract}
The Virtual Learning Environments (VLE) are considered virtual sites that have a vast amount of resources that allow it to host courses that occur as Distance Education (EaD in Portuguese) or blended mode, favoring communication between the actors involved in these arrangements. Assign Artificial Intelligence to VLEs using Multi-Agent Systems (MAS) is a way to facilitate the learning process. By analyzing the state of the art of existing VLEs today, it can be seen that they all work as tools to aid students, but none works the management aspects of distance education supporting legislation. Therefore, this article presents a model and VLE-MAS integration system that can make the VLE able to assist managers of distance education in their tasks incorporating legislation representation. In addition, this system will work in an evolutionary way where the machine-learning method called the nearest neighbors algorithm will be applied to help the dead-time warning mechanism.
\end{abstract}

Keywords: multi-agent systems, organizational models, virtual learning environments, distance education, legal systems.

\section{$1 \quad$ Introduction}

In many countries, such as Brazil, Distance Education (EAD), has become a chance to democratize education, allowing social inclusion, as it can reach places where there are no educational institutions (EIs).

In Brazil, distance education is governed by legal bases that were established by the Law of Guidelines and Bases of National Education (Law no. 9,334). The legislation that supports Distance Education also regulates and determines its actions and functions, while one of the great challenges is to make the EIs meet the educational requirements, ensuring safety and quality throughout the educational process developed in this mode. 
The technology that enabled the advancement of distance education was the development of focused virtual spaces to the mode called Virtual Learning Environments (VLE).

In view of the process that involves administrative nature issues, existing VLEs today, do not have any support, as well as work in a distanced way from laws, rules and regulations that the government and EIs determine for the proper conduct of distance education. Thus, VLEs are unable to enforce their players the rules that are in force, affecting the actions carried out in the environment to those standards.

This paper proposes an alternative to that deficiency in the support to legislation in the existing VLE to enable the incorporation of laws and regulations governing distance education, aiming to help on tasks related to institutional management. Based on this, the objective of this article is to present the creation of a system based on the model that allows the integration to VLE MOODLE of the main aspects of distance education legislation, intermediated by an Multi-Agent System (MAS).

One way to manipulate the interaction of agents in an MAS is the use of an organizational structure and we used an adaptation of the Moise+ model that will define the behavior of the actors in distance education process, regulating the restrictions and permissions that are set by laws and regulations [2]. In order for the model to be able to make some decisions based on event and/or actions previously performed by agents that make up society, the MAS will be applied alongside data mining techniques, making the model able to recognize some patterns displayed by the VLE user, and from that make decisions.

For the formalization of this model, the modality of distance education in a Federal Institute (FI), will be used as a case that currently offers higher education, technical and continuing education programs in the distance mode, focusing on a government program called Network e-Tec, which focuses on the provision of technical courses and the initial and continuing training of workers.

\section{Multiagent Systems and their Organizational Models}

The MASs allow for intelligent global behavior that can be achieved through the individual behavior of agents that compose it, based on the social behavior of a community of independent agents.

To [1], an organization is defined as a pattern that describes how the members belonging to a society are in relation to others and how they interact to achieve a common goal. To organize an MAS, it is necessary to work with independent agents, as achieving a global goal requires that autonomy offers limits. The MAS organization is a set of restrictions on the behavior of agents in order to lead to a common goal. There are several types of organizational models, but the model chosen for the development of the work was the Model of Organization for Multi-Agent+ (Moise +), developed by [2] because of its ease of incorporation into the Jason platform [3].

Moise+ considers that the goal of an organization is to restrict the agent's autonomy. 
The restrictions expressed in Moise+ are indicated by the requirement of permission and prohibition. It also works with the organization of an MAS based on three dimensions, shown below.

The structural specification (SS), is based on three main concepts [2]: 1) roles: it is a set of behavioral constraints that an agent agrees to when joining a group; 2) bonds and compatibilities: the roles are related to others representing restrictions on interactions; 3 ) groups: represent a set of agents with higher affinity and close goals.

The functional specification (FS), consists of a set of social schemes, and a preferred ratio between missions. A social scheme is based on the idea of global targets; whose function is to represent a world state that is desired by the MAS. The main concepts related to the global targets are: missions: is a set of global targets that can be assigned to an agent through their roles; social scheme: a decomposition tree of global targets where the root is the goal of a social scheme; preferences between missions: in some cases, it is necessary to establish an order of preference between missions. The deontic specification (DE), aims at relating the SS with FS, establishing which tasks a role is allowed or required to commit to.

\section{Kelsen's Legal System Concept}

Incorporating the MAS to VLE as well as working with the normativity within social systems is still an existing challenge. But using these normative notions such as duty, obligation and ban facilitate the design and the computer systems specification, making them more autonomous. Based on this question, it is essential to present the issues related to normativity proposed by Hans Kelsen.

The starting point of the theory proposed by Kelsen is the idea of norm as something that determines an objective sense of must-do, highlighting the imperative character, both as imposition and ban of this must-do [5].

A rule determines how to behave in certain conditions, i.e., if a behavior and social relationships may be prescribed, prohibited or permitted. A rule indicates either conduct or omission of conduct that should be avoided by individual agents of the legal system.

According to [5], the functions of the rule of law are divided into standards: imposing, permission, authorization and derogation. The legal standards form a regulatory system that requires that those involved are in accordance with the modes of behavior stated in the rules. Each standard is derived from a higher standard. The base system is a fundamental rule of law. And a legal system that consists of a hierarchy of standards and a set of agents who supervise compliance with the rules.

\section{4 k-Nearest Neighbors Algorithm}

According to [6], the k-Nearest Neighbors algorithm (k-NN) is a powerful nonparametric algorithm used in data mining for classification and pattern recognition.

The k-NN requires a set of pre-classified examples. Based on this set of examples, a new pattern $\mathrm{K}$ of unknown class is compared to the set of pre-classified examples 
through the relationships between attributes, that is, the $\mathrm{K}$ classifier seeks $\mathrm{K}$ elements from a set of examples nearest this unknown element for the shortest distance.

Thus, it is necessary to classify each new instance and seek $\mathrm{K}$ instances with the shortest distance in relation to this new instance.

There are different ways to calculate this distance, the simplest of which is the Euclidean distance (1), where pi is the value of the current line $i$ that one wants to discover the nearest neighbor; qi is the value of each row in column $i$; and, maxi and mini are respectively the maximum and minimum value of all column i:

$$
d(p, q)=\sqrt{\sum_{i=1}^{n}\left(\frac{\left(p_{i}-q_{i}\right)}{\left(\max _{i}-\min _{i}\right)}\right)^{2}} .
$$

\section{Organizational Model for a Legislation-Oriented VLE-MAS}

This section aims at presenting an organizational model for VLE-MAS, based on the FI case study, to be able to integrate legal aspects, making the Legislation-Oriented VLE-MAS. To formalize this model, we will use a variation of the organizational model Moise+ by the fact that this model is able to restrict the behavior of agents.

\subsection{Actors of Distance Education Mode and Structural Specification (SS)}

In the FI concerned, connected to the Vice President's Office of Education, there are the actions involving distance education, which currently perform the planning and regulation of the activities of this type. Connected to the Vice President's Office, we have the campus of the FI, with its distance education departments, composed of various actors, such: Course Coordinator who is a teacher with training in related field to the course and responsible for its management; Research Professor: it is one whose role is to plan and manage all the learning development process in the subject and, Distance Tutor: will monitor the activities carried out online by students.

Based on these actors, we propose the creation of the SS of the VLE-MAS, where the roles, groups and relations between them will be presented. In distance education, we have several agents committed to the abstract role of coordinator. These are: a center, course, tutoring, general or deputy general coordinator. Agents committed to the teacher abstract role can be a content developer or research professor. The agents who assume the abstract role of the tutor can be divided into: classroom tutor and distance tutor.

In addition to these roles, there are a set of roles that take a collective level thus forming groups. Finalizing the organizational modeling of SS, in relation to the campus we have the following groups: Coordination of distance education, Center, IT Team. 


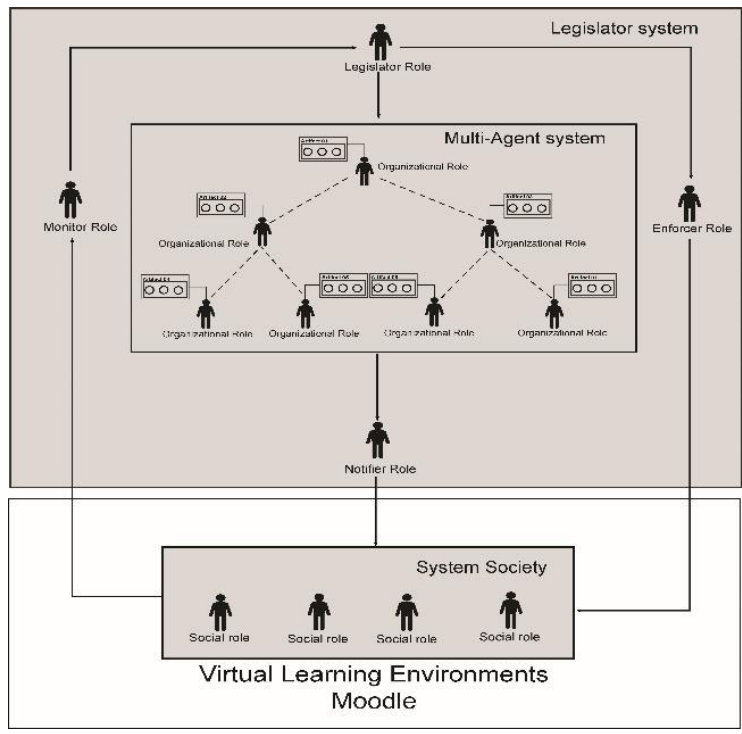

Fig. 1. Legislation-Oriented VLE-MAS System Model.

\subsection{Functions and Functional Specification (FS)}

Each role has a set of functions that are legislated by Law. 9.394/1996. Because of the great diversity of function, this article will present the definitions only of the role of the Course Coordinator, whose responsibilities are, for example: follow the course; carry out the academic management of the classes; monitor the activities of tutors, teachers; in-loco verification of the progress of the courses.

With these defined functions, we can determine the FS, with a set of global goals, structured in a goal decomposition tree, which is made by plans showing a way to meet this goal. In this case the tree root is the goal to be achieved and the stipulated missions determine the order in which the plans must occur so that they can achieve the specific objective.

\subsection{Contractual Rules and Deontic Specification (DS)}

Based on the internal rules of the FI and the contractual rules, the program can define the duties, rights, obligations to be performed by the Course Coordinator.

The rights of a course coordinator are: access to VLE-MAS; participate in training and updating activities. While the duties of this player include holding the record of the assessments undertaken by students; coordinating the preparation of the course of the project; follow the academic activities of the course. With the contractual standard, we can define the DS where we have the definition of the role will be responsible for carrying out the actions, the name of the actions, the description of the goals and legislation where it is found. While the deontic relation of this model is stipulated by 
three concepts: duty, law and the prohibition of the mission. Finally, we have time restriction, because the deontic relations must have a shelf life. In addition to that, two columns were inserted, which determine which roles will monitor the action and apply punishments and who should help so the action can reach its goal.

\section{Legislation-Oriented VLE-MAS System Model}

To model and develop the system of the legislation-oriented VLE-MAS some fundamental aspects of systematic, hierarchical model of distance education of the FI were taken as a base, and the instrumental approaches determined by the Ministry of Education. The proposed legislation-oriented VLE-MAS system is composed of two subsystems, as shown in Figure 1, where the components, its organization and its functions will be detailed below.

\subsection{Multi-Agent System}

The multi-agent system is integrated within the VLE, and therefore uses its database with users and profiles that are registered.

The multi-agent system allows users to make their actions usually so the legislature system can monitor and verify the legality of such activities. It can be said that part of the system is the VLE itself. Within the multi-agent system, we have various roles called Social Agents without hierarchy. These social agents that make up the system take on roles or group roles. In this case, the system is divided into various categories, and a user can assume different roles, where, for example, a teacher may be a member of the multidisciplinary team or the teacher who will teach a course.

\subsection{Legislator System}

The legislator system will aim at monitoring all actions performed in the system of society in order to ensure that agents are acting within the law. If social roles are not fulfilling their goals, the system is responsible for sending notifications and applying penalties.

Still within the legislative system, a multi-agent system will be included based on an organizational model previously introduced that will work with the hierarchy of roles or role groups.

The legislator system will consist of four main roles that are to be regarded as software agents, which will interact with each other in order to monitor and evaluate the progress of actions and goals that are taking place within the company system.

The first part is called the legislator role, which is responsible for carrying out the actions to create, modify and remove rules and plans of the MAS, and is considered an issuer of standards. In this case, the legislative role will be sovereign over the rules, therefore, all tasks to create, modify and remove rules is the responsibility of this role.

The second part is called the monitor role, whose purpose is to assist in the extraction and collection of information of what is happening in the company system. This agent 
is watching real-time system actions to see if the other agents are complying with the rules and plans.

The monitor roles can be classified into two types: 1) Standard Monitors: observe the standards and behavior of social roles, capturing information regarding compliance or not with those standards; 2) Plan Monitors: capture information about the current state of social roles, ensuring the implementation of plans to be undertaken to achieve the goals. In this case, the legislature system may have at least two monitoring agents.

The third role is a notified role, which aims at presenting reminders (via VLE block and email), of the outstanding actions or deadlines to be met before being penalized. In addition to that, this role will also have the purpose of showing where the rules were based upon, that is, which law, rules, regulations or contracts the rules were based upon and consequently extracted. In this case, the model will be based on deontic specification of the MAS in order to get this information. The notified also based on registered email in VLE, will trigger messages to users about pending or penalties applied to agents. Finally, the last agent is called Enforcer role, and its function is to assist in the implementation of standards and plans and will be responsible for applying the sanctions and penalties when the rules are not followed correctly.

The Enforcer role will have two subdivisions: Penalties Enforcer performing the effective implementation of sanctions and penalties prescribed in the rules of the social roles that violate it and the plan Enforcer, performing the action plans and altering the structural properties of the set of social roles. While the MAS roles, which is located within the legislative system will be the same roles used in VLE, which in this case work with the notion of hierarchy. This hierarchy was determined according to the SS of the organizational model.

This hierarchy shows the roles according to their order of autonomy and is based on certain rules that are important to that society agents where the role with lower position is always subordinate to roles with top positions. In this case, the roles are no longer called social roles to be called organizational roles that become proactive components of the legislative system, because they are linked to a set of artifacts and its main feature is autonomy.

These devices are based on the metamodel of Agents and Artifacts (A\&A), [4] which incorporates a set of goals and plans. Thus, it can be said that these artifacts are the laws, rules, regulations, decrees and agreements that will determine the actions to be performed by the agents and other roles.

\section{Development of the Legislation-Oriented VLE-MAS System Applied to Moodle}

Initially it was necessary to conduct a research on the MOODLE main structure as well as the development of new blocks of rules. It can be seen that MOODLE is divided into two structural parts called standard and development structures.

The first structure is composed of the basic features that the environment offers, which include course management tools, user authentication and commonly used 


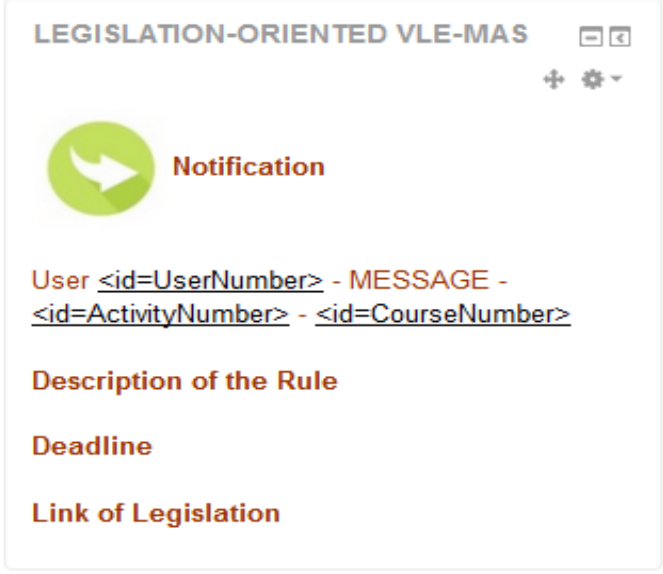

Fig. 2. Components of the Message.

functions. While the development structure consists of modules and auxiliary blocks, which have the ability to be turned on or off as needed.

Some of these blocks come together with MOODLE, but others can be developed using the programming language PHP 5 (Hypertext Preprocessor). Thus, we can say that the legislation-oriented VLE-MAS system is an example of blocks that can be developed by users.

From version 2.7, MOODLE provides a module called "Local" that enables the transformation of MOODLE in a complete development framework. Thus the legislation-oriented VLE-MAS system is being developed within the MOODLE API itself, in version 3.1, also making necessary the use of PostgreSQL.

In order to facilitate the implementation of MOODLE is taking as a basis the use of an existing MOODLE plug-in called blog, which has access to the VLE database.

\section{Partial Prototype of the Legislation Oriented VLE-MAS System Applied to Moodle}

The legislation-oriented VLE-MAS system when applied to MOODLE [7] notifies users of the company system through a box built into the environment called box that can be considered a MOODLE plug-in. When you activate this plug-in, the Monitor Role will review all user actions based on rules previously established by the Legislator Role and in the actions that must be performed by the system in case of compliance or non-compliance with these rules. By identifying a rule pending or that has been met, the Monitor Role in conjunction with the Notified Role will display a message box formed by the components shown in Figure 2.

In addition to that, the Monitor Role will review all actions that are not held and the Enforcer Role will conduct sanction actions on users of the system of society that fail to comply with the rule, within the stipulated limit. 
The Enforcer Role always shows a message to the box on the action that was taken back to higher users as deontic specification of the organizational model MAS, as well as a message via email. The Enforcer message is composed of: User name and default message for each action performed by the Enforcer role.

To perform these simulations, the interface implementation was used, using the PHP language, built into MOODLE version 3.1. The choice of VLE MOODLE 3.1 was due to the fact that all of the FI's distance education programs in question already use it. Based on this interface simulation, the following will be presented as an example of how MOODLE behaves with regard to compliance or non-compliance with certain rules involving the course coordinator.

A course coordinator to be selected to perform the role should make a previous registration in MOODLE. After that, he/she should post on MOODLE supporting documents to work in the role.

Figure 2 shows a diagram representing the exchange of messages held by users as well as the actions taken by the Enforcer role for the CompExp Rule.

By posting, the General Coordination will get through the Notifier role a warning that all documents have been posted. In addition to that, the manager who is hierarchically above the Course Coordinator, in case of Department Coordinator also receives a notification.

Thus, General Coordination makes manual conference documents. If the documents are all delivered with no pending issues, the Notifier role will send a return message to that user while the Enforcer Role releases access in all areas of MOODLE, with the visitor user being promoted to Course Coordinator. In the case of the documents presented including dependencies, the multidisciplinary team will mark which documents have problems choosing from a list what is pending according to the legislation. Also, it is given a period for resending the document.

An example of pending documents can be when the course coordinator does not have the certificate attesting teaching experience required by law. In this case, the multidisciplinary team, led by general coordinator and assistant, chooses in the list that the rule is pending. After this Notified role will send both the course coordinator and the department a message with the user's name, pending legislation as well as a link to the law governing this rule and the deadline for the new posting of the document.

If the document is returned on time and is in agreement, the Notified role will send a return message to that user while the Enforcer Role releases access in all areas of MOODLE. If the outstanding documents are not submitted on time and/or not in accordance with the law, the Notified role will cut user access and notify the roles that are hierarchically above the role of this action.

\section{Application of the k-nn Algorithm to the MAS-VLE Model}

In order for the proposed model to be capable of autonomously deciding when to show delay messages to each of the users, the k-NN algorithm was implemented through a virtual learning environment (VLE), multiagent system (MAS). 
The purpose of applying the algorithm to the VLE-MAS is to prompt the system to analyze similar actions previously performed by users and, based on this analysis, define the time that current users need to perform the same action. As such, the notified role will only display messages if the user exceeds the time determined by the k-NN algorithm.

For example, the VLE-MAS system along with the k-NN algorithm analyze all the courses in the VLE in order to determine the time each professor takes to mark an activity. Based on the number of posts, number of objective and subjective questions, class hours and type of activity, the K-NN algorithm determines the nearest neighbors, that is, the time taken by other professors to mark similar activities. Should the professor exceed this time, the notified role will advise that there are $\mathrm{X}$ days left to mark it or that marking is $\mathrm{X}$ days late.

\subsection{Stage 1 - Preparing the Training Data Set}

In order to test the operation of the algorithm developed in PHP and applied to the VLEMAS, a data set important to the functioning of the k-NN was extracted from the database of the Federal Institute`s (FI) MOODLE VLE. The data extracted were:

- Professor's name;

- Class hours for the course (HRS);

- Number of activities per course (NA);

- End date for posting, that is, the latest date on which students can hand in their activities, since the professor can only begin marking after this date;

- Date of marking completion, that is, the last day on which the professor marked the activities;

- Type of activity, namely 1 for forum, 2 for questionnaire, 3 for sending a file and 4 for glossary (TA);

- Number of objective questions (NOQ);

- Number of descriptive questions, which take longer to be marked (NDQ);

- Number of attempts, that is, the number of students who sent activities to be marked (NATT);

- Whether marking occurred within the deadline or not, using 1 when the deadline was exceeded and 0 when it was not. To that end, teaching guidelines stipulating that professors have 15 to 20 days to mark activities was applied (Deadline);

- Number of days the professor took to mark the activity based on the end date for posting and date of marking completion.

\subsection{Stage 2 - Example 01 - Obtaining the Nearest Neighbors}

Using the K-NN algorithm applied to the VLE-MAS, testing was conducted with the following data set (Table 1).

The aim is to discover how many days' professor B would take to mark activity 6. To that end, the following formula was applied (2): 
Table 1. Data set - Example 01 .

\begin{tabular}{|c|c|c|c|c|c|c|c|c|c|c|}
\hline Teacher & Hrs & NA & $\begin{array}{c}\text { Posting } \\
\text { End Date }\end{array}$ & $\begin{array}{c}\text { Marking } \\
\text { Completion } \\
\text { Date } \\
\end{array}$ & $\mathbf{T A}$ & NOQ & NDQ & NATT & Deadline & Days \\
\hline A & 30 & 1 & $16 / \mathrm{mar} / 15$ & $23 / \mathrm{mar} / 15$ & 3 & 0 & 1 & 340 & 0 & 7 \\
\hline A & 30 & 2 & $16 / \mathrm{mar} / 15$ & $21 / \mathrm{mar} / 15$ & 3 & 0 & 1 & 338 & 0 & 5 \\
\hline A & 30 & 3 & $16 / \mathrm{mar} / 15$ & 06/apr/15 & 3 & 0 & 3 & 284 & 1 & 21 \\
\hline A & 30 & 4 & $16 / \mathrm{mar} / 15$ & $27 / \mathrm{mar} / 15$ & 2 & 10 & 0 & 306 & 0 & 11 \\
\hline $\mathrm{B}$ & 60 & 1 & 22/apr/15 & $11 /$ may/15 & 4 & 0 & 1 & 306 & 1 & 19 \\
\hline $\mathrm{B}$ & 60 & 2 & $16 / \mathrm{mar} / 15$ & 29/apr/15 & 3 & 0 & 10 & 300 & 1 & 44 \\
\hline $\mathrm{B}$ & 60 & 3 & $23 / \mathrm{mar} / 15$ & $31 / \mathrm{mar} / 15$ & 1 & 0 & 1 & 338 & 0 & 8 \\
\hline $\mathrm{B}$ & 60 & 4 & $25 / \mathrm{mar} / 15$ & 06/apr/15 & 2 & 0 & 5 & 217 & 0 & 12 \\
\hline $\mathrm{B}$ & 60 & 5 & 06/apr/15 & 18/apr/15 & 3 & 0 & 1 & 242 & 0 & 12 \\
\hline $\mathrm{B}$ & 60 & 6 & 06/apr/15 & 24/may/15 & 3 & 0 & 1 & 338 & 1 & ?????? \\
\hline
\end{tabular}

Table 2. Score - Example 01.

\begin{tabular}{c|c|c}
\hline Teacher & Score & Days \\
\hline A & 1.732127 & 7 \\
\hline A & 1.624808 & 5 \\
\hline A & 1.262039 & 21 \\
\hline A & 1.829971 & 11 \\
\hline B & 1.085724 & 19 \\
\hline B & 1.24316 & 44 \\
\hline B & 1.343296 & 8 \\
\hline B & 1.548824 & 12 \\
\hline B & 1.284197 & 12 \\
\hline B & 0 & $? ? ? ? ? ?$ \\
\hline
\end{tabular}

$$
\sqrt{\left(\frac{(60-H R S)}{(60-30)}\right)^{2}+\left(\frac{(6-N A)}{(6-1)}\right)^{2}+\left(\frac{(3-T A)}{(4-1)}\right)^{2}+\left(\frac{(0-N O Q)}{(10-0)}\right)^{2}+}
$$

At the end, a score was generated for each line of the data set (Table 2).

The scores were used to determine the shortest distance, that is, the lowest score was 1.085724, meaning that the average time Professor B took to mark the activities was 19 days. Should he exceed this number, the notified role should send a message to that effect.

At each execution, the data set is applied to the KNN algorithm and thus always updated at the end of each period, making its execution based on an evolutionary 
prediction. Throughout each run, new samples are incorporated into the data set making the algorithm more accurate, causing SCORE to change with each run.

\section{Final Considerations}

The legislation-oriented VLE-MAS system was developed in order to assist the manual work done today by distance education managers when working with issues related to corporate or institutional management process. With the proposed model and the system, it is being possible to trace directions for automation of control roles in relation to legislation, since it became possible to carry out the systematization of knowledge about actions and plans that agents must perform.

The advantage of the model, to be incorporated into a VLE interface, allows any user who has already received training to use the model without major difficulties, since the design and simplicity of the environment allows easy and enjoyable use that leads to understanding and carrying out of actions whose legal constraints are often considered difficult to understand. In future projects, you can implement a greater number of actions as well and incorporate part of reports available to managers, with activities involving administrative matters carried out or not by users of the system of the society.

\section{References}

1. Fox, M.: The Tove project towards a common-sense model of the enterprise. Proceedings of the 5th International Conference on Industrial and Engineering Applications of Artificial Intelligence and Expert Systems, Springer-Verlag, pp. 25-34 (1992)

2. Hübner, J. F., Sichman, J. S., Boissier, O.: Moise+: Towards a structural, functional, and deontic model for mas organization. Proceedings of the First International Joint Conference on Autonomous Agents and Multiagent Systems, (ACM), pp. 501-502 (2002)

3. Boissier, O., Bordini, R., Hübner, J. F., Ricci, A., Santi, A.: Multi-agent oriented programming with JaCaMo. Science of Computer Programming, pp. 747-761 (2013)

4. Hubner, J. F., et al.: Instrumenting multi-agent organisations with orgnisational artifacts and agents. Autonomous Agents and Multi-Agent Systems, Kluwer Academic Publishers, Hingham, 20(3), pp. 369-400 (2010)

5. Kelsen, H.: Teoria Pura do Direito (2009)

6. Mitchell, T.: Machine Learning. McGraw-Hill (1997)

7. Dougiamas, M., Taylor, P. C.: MOODLE: Using learning communities to create an open source course management system. Proceedings (EDMEDIA) (2003) 